
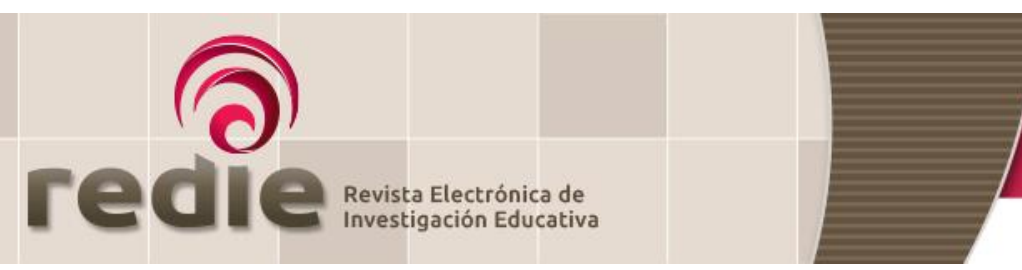

\title{
Adaptación de las escalas de expectativas de resultado, metas de progreso y satisfacción académica en estudiantes universitarios
}

\section{Adaptation of Outcome Expectations, Progress Goals and Academic Satisfaction Scales in College Students}

\author{
Mauricio Federico Zalazar-Jaime (*) mfzalazar@gmail.com \\ Marcos Cupani (*) marcoscup@gmail.com \\ (*) Instituto de Investigaciones Psicológicas (CONICET y UNC) \\ (Recibido: 28 de octubre de 2016; Aceptado para su publicación: 07 de marzo de 2017)
}

Cómo citar: Zalazar-Jaime, M. F. y Cupani, M. (2018). Adaptación de las escalas de expectativas de resultado, metas de progreso y satisfacción académica en estudiantes universitarios. Revista Electrónica de Investigación Educativa, 20(3), 105-114. Recuperado de https://doi.org/10.24320/redie.2018.20.3.1675

\section{Resumen}

La deserción universitaria es una de las problemáticas que afectan a las instituciones de educación pública en Argentina. Considerando que no existen medidas específicas para evaluar las variables centrales del modelo de persistencia académica propuesto por la teoría Social Cognitiva, el objetivo de este trabajo fue adaptar las escalas de expectativas de resultado, metas de progreso académicas y satisfacción académica en una muestra de estudiantes universitarios. En el estudio participaron 1,282 estudiantes de diferentes carreras en la Universidad Nacional de Córdoba (Argentina). En líneas generales, las propiedades psicométricas fueron adecuadas (estudios de consistencia interna y evidencia de estructura interna). No obstante, no se pudo replicar la relación entre las expectativas de resultado y las metas de progreso.

Palabras clave: Expectativa educativa, calidad de la educación, satisfacción académica; deserción escolar, educación superior.

\section{Abstract}

Dropout is a problem for public education institutions at university level in Argentina. Considering there are no specific measurements to evaluate core variables in the academic persistence model proposed by social cognitive theory, the objective of this study was to adapt the outcome expectations, academic progress goals and academic satisfaction scales in a sample of college students. The study included 1,282 students from different degree programs in the National University of Córdoba (unc; Argentina). Generally speaking, the psychometric properties were adequate (with tests for internal consistency and evidence of internal structure). However, it was not possible to replicate the relationship between outcome expectations and progress goals. 


\section{Introducción}

La Teoría Social Cognitiva de la Carrera (scct, por sus siglas en inglés) (Lent, Brown y Hackett, 1994) es una de las propuestas que mayor atención ha recibido en el campo de la psicología educacional (Flores, Navarro y Ali, 2016). La SCCT consta de diferentes modelos interrelacionados, los cuales intentan explicar cómo las personas desarrollan sus intereses vocacionales, seleccionan una carrera, alcanzan determinados niveles de rendimiento/persistencia (Lent et al., 1994), experimentan bienestar y disfrute en las actividades académicas (Lent y Brown, 2006; 2008) y se adaptan a diferentes aspectos involucrados en el proceso de carrera (Lent y Brown, 2013).

Independientemente del comportamiento que se intente explicar, el núcleo central de la scct está representado por los lineamientos propuestos por la teoría Social Cognitiva (TSC) desarrollada por Bandura (1987). Particularmente, la TSc destaca el carácter activo del individuo y la interacción de éste con factores personales, contextuales y ambientales en la manifestación de la conducta. En este sentido, Bandura (1987) establece que las creencias de autoeficacia, expectativas de resultado y metas permiten entender, por un lado, la forma en que el individuo procesa e interpreta cognitivamente los estímulos y, por otro, actúan mediando la relación entre variables personales (por ejemplo, género y rasgos de personalidad) y factores contextuales (Sheu y Bordon, 2016).

Bandura (1987) define la autoeficacia como las creencias que poseen las personas sobre sus capacidades para organizar y ejecutar las acciones que les permitan lograr un rendimiento exitoso en dominios específicos. Dicho constructo ha sido uno de los más abordados en la literatura (Maddux, 2012) debido que afecta la forma en que las personas piensan, se comportan y se motivan a sí mismas (Bandura, 1997). Sin embargo, no es suficiente contar con adecuadas creencias de autoeficacia, también es necesario orientar el comportamiento con base en ciertos fines (Fouad y Guillen, 2006). En este punto, las expectativas de resultado refieren a las creencias respecto a las posibles consecuencias de realizar ciertas conductas (por ejemplo, "si intento hacer esto, ¿qué pasaría?"). Bandura (1987) sostiene que las consecuencias pueden ser físicas (por ejemplo, monetaria), sociales (aprobación o aliento) y autoevaluativas (satisfacción), y el contenido de cada uno puede referirse a eventos positivos, negativos o neutros.

Por otro lado, las metas operan a través de la capacidad de representar simbólicamente los resultados deseados en el futuro, y estos determinarán la participación en una actividad en particular, incluso durante largos períodos de tiempo aun ante la falta de reforzadores. Adicionalmente, la scct distingue dos tipos de metas, la primera referida al dominio (o actividad) que se pretende realizar (metas de elección), y la segunda vinculada al nivel de rendimiento hacia el cual el sujeto aspira en un dominio dado (metas de rendimiento) (Lent et al., 1994); ambas modalidades son importantes reguladores de la conducta y actúan de manera diferencial, la primera motiva a los estudiantes a perseguir sus opciones educativas/profesionales, y la segunda, estimula para alcanzar determinados estándares de desempeño en tareas específicas.

Si bien varios estudios desarrollados bajo el marco de la scct han incluido las expectativas de resultado, éstas han sido operacionalizadas de diferentes modos (Fouad y Guillen, 2006). Inicialmente se utilizó una versión adaptada de la escala valor-expectativa del modelo de motivación laboral desarrollado por Vroom (1964). La misma presentaba diferentes valores (recompensa, esfuerzo y motivación, entre otros), donde los participantes debían contestar mediante dos escalas de respuesta, una que indicara la importancia (de "nada importante" a "muy importante") y otra, la probabilidad (de "nada probable" a "totalmente probable") de satisfacer cada uno de esos valores. Sin embargo, esta modalidad presentó ciertas dificultades en la estimación de los puntajes, por tanto, desarrollos posteriores contemplaron escalas de respuesta donde los sujetos sólo debían indicar la probabilidad o acuerdo de que se produzcan determinados resultados (Fouad y Guillen, 2006).

Otra serie de estudios han utilizado versiones modificadas de la escala Expectativas de Resultado en Matemáticas y Ciencias desarrollada por Fouad y Smith (1996), quienes entienden las expectativas como aquellos comportamientos específicos que podrían ser útiles en el futuro. Sin embargo, la escala de 
Expectativas Académicas de Resultado desarrollada por Lent et al. (2005) ha sido una de las más utilizadas en la literatura educacional y, además, retoma los lineamientos originales propuestos por Bandura (1987). Esta escala refiere a las posibles consecuencias positivas producto de terminar una carrera de grado e involucra implícitamente diferentes valores como prestigio (conseguir el respeto de otras personas), compensación monetaria (ganar un buen sueldo) y autoevaluación (aumentar la autoestima).

Respecto a las metas, éstas han sido evaluadas solicitando a los participantes que indiquen su intención de aspirar a realizar una actividad (Lent y Brown, 2006). Particularmente Lent et al. (2005) desarrollaron una escala (metas de progreso) para evaluar el avance de los estudiantes en sus estudios (por ejemplo, mantener notas altas en todas las materias). Si bien dicho instrumento refiere a la carrera académica en general y no especifica un área de conocimiento en particular, otros trabajos han adaptado esta escala para estudiantes de ingeniería (Lent, Singley, Sheu, Schmidt y Schmidt, 2007) y metas relacionadas al trabajo (Duffy y Lent, 2009), entre otros.

En la literatura se han corroborado las principales hipótesis de la TSC respecto a la relación entre las creencias de autoeficacia, expectativas de resultado y metas (Lent, 2005; Rottinghaus, Larson y Borgen, 2003). Asimismo, Lent (2004) postula que dichas variables contribuyen a explicar la satisfacción académica, la cual refiere al bienestar y disfrute que los estudiantes perciben al llevar a cabo experiencias relacionadas a su rol como estudiantes (Lent y Brown, 2008). Se ha evidenciado que la satisfacción académica constituye un factor importante debido a que se relaciona con el ajuste académico, la integración social y la persistencia académica (Lent, Taveira, Sheu y Singley, 2009; Lent et al., 2007; Lent et al., 2013). Por tanto, Lent et al. (2005) desarrollaron una escala para evaluar el nivel de satisfacción, tanto general ("estoy contento/a con mi vida académica") como específico ("disfruto el nivel de estimulación intelectual de cada temática") de los estudiantes, la cual ha reportado adecuadas propiedades psicométricas.

Como destaca el Instituto Internacional para la Educación Superior en América y el Caribe (2006), en las últimas décadas el porcentaje de estudiantes de nivel superior que abandona sus estudios ha aumentado paulatinamente. En Argentina, según las cifras oficiales del año 2010, se observó que la deserción universitaria ascendió al 59.6\% (Secretaría de Políticas Universitarias [SPU], 2010). A pesar de que la SPU no ha continuado con la medición de este fenómeno, algunos trabajos (García de Fanelli, 2013; Parrino 2012) han indicado valores similares y/o superiores a los reportados previamente. García de Fanelli (2011), por ejemplo, destaca que "de cada 100 inscriptos en las universidades nacionales, egresa un promedio de 22 estudiantes" (p. 12). Es importante destacar que si bien cada trabajo ha establecido diferentes criterios para la estimación del abandono universitario, las cifras son preocupantes.

Por tanto, considerando que en nuestro medio no existen medidas específicas y estandarizadas que permitan evaluar las variables centrales del modelo de rendimiento/persistencia académica propuesto por la scct, el objetivo de este trabajo consistió en adaptar las medidas de expectativas de resultado, metas de progreso y satisfacción académica en una muestra de estudiantes universitarios argentinos.

\section{Método}

\subsection{Participantes y procedimiento}

En el estudio participaron 1,282 estudiantes de la Universidad Nacional de Córdoba (UNC, Argentina): 932 (72.7\%) del sexo femenino y 314 (24.5\%) del sexo masculino (36 participantes [2.8\%] no brindaron respuesta respecto al sexo), con edades comprendidas entre los 17 y 65 años $(M=22.08 ; \mathrm{DE}=5.96)$. Del total de la muestra, 382 participantes se encontraban realizando el curso de ingreso a la facultad de Psicología (UNC), mientras que los restantes 900 alumnos cursaban otras carreras en la misma universidad distribuidas: Ciencias Aplicadas 12.1\%, Ciencias básicas 2.8\%, Ciencias de la Salud 20.1\%, Ciencias Humanas $14 \%$ y Ciencias Sociales 3.5\%. La administración de los cuestionarios se desarrolló de forma colectiva y en horario regular de clases. Previo a la administración se explicó el objetivo del estudio en lenguaje claro y comprensible, se destacó la libre voluntad de participar y la confidencialidad de la información obtenida. 


\subsection{Instrumentos}

Expectativas Académicas de Resultado (Lent et al., 2005). Esta escala está compuesta por 10 ítems que indagan sobre los posibles resultados de la graduación. En un estudio desarrollado por Lent et al. (2005), los autores reportaron una estructura factorial compuesta por dos factores: expectativas académicas intrínsecas (relacionadas con experiencias subjetivas tales como el interés y satisfacción) y extrínsecas (consecuencias reforzantes externas o tangibles, tales como el dinero y el respeto). Los participantes deben responder utilizando una escala tipo Likert con 10 opciones de respuesta, donde 0 representa "Totalmente en desacuerdo" y 9 "Totalmente en acuerdo". En su versión original esta escala cuenta con estudios de consistencia interna (alfa de Cronbach) de .90.

Metas de Progreso Académicas (Lent et al., 2005). La escala está compuesta por 7 ítems e indaga el progreso académico que se de los estudiantes en temáticas como aprendizaje y comprensión de la currícula universitaria. Los participantes deben responder mediante una escala tipo Likert con 5 opciones de respuesta, desde 1 "Ningún progreso" a 5 "Excelente progreso". Respecto a las propiedades psicométricas, este instrumento ha reportado resultados satisfactorios de consistencia interna (alfa de Cronbach) de .86 .

Satisfacción Académica (Lent et al., 2005). Esta escala está compuesta por 7 ítems que indagan sobre el nivel de satisfacción de los estudiantes respecto a su experiencia académica. Los participantes deben responder usando una escala tipo Likert con 5 opciones de respuesta, donde 1 representa "Totalmente en desacuerdo" y 5 "Totalmente de acuerdo". En su versión original este instrumento cuenta con estudios de consistencia interna (alfa de Cronbach) de .87, y evidencia de validez predictiva.

\subsection{Estrategia de análisis}

Mediante el software SPSS se evaluó el patrón de valores perdidos, considerando los ítems de cada instrumento con el fin de estimar si el mismo respondía a una distribución aleatoria (Tabachnick y Fidell, 2011). Se calculó la media, desviación estándar, asimetría y curtosis. Como criterio para evaluar los índices de asimetría y curtosis se consideró como excelente valores entre \pm 1.00 , y adecuados valores inferiores a \pm 2.00 (George y Mallery, 2011). Se identificaron los casos atípicos univariados mediante el cálculo de puntuaciones Z (puntuaciones Z > \pm 3.29 fueron considerados atípicos), y multivariados mediante la prueba de distancia de Mahalanobis $(p<.001)$.

Posteriormente se realizó un Análisis Factorial Confirmatorio (AFC) con el fin de replicar las estructuras originales de cada instrumento. Para ello, se utilizó el software estadístico Mplus versión 6.12, el cual permite aplicar el método de estimación denominado mínimos cuadrados ponderados (WLS, por sus siglas en inglés), considerado el más oportuno cuando se trabaja con datos categóricos (Flora y Curran, 2004). Para evaluar el ajuste se utilizaron los estadísticos Chi-Cuadrado, el Índice de Ajuste Comparativo (CFI), el Índice de Tucker-Lewis (TLI), el Error Cuadrático Medio de Aproximación (RMSEA), y la Media Cuadrática Residual Ponderada (WRMR). Los valores entre .90 y .95 o superiores para el CFI y TLI son considerados como ajustes aceptables a excelentes, se esperan valores entre .05 y .08 para el RMSEA, y valores menores a 1.00 para WRMR (Yu y Muthén, 2002).

Se estimó la consistencia interna mediante el estadístico de confiabilidad compuesta ( $\rho$ ), el cual ha sido reportado por la literatura como el más adecuado, en comparación al coeficiente alfa de Cronbach, debido a que presenta una menor variación del error (Cortina, 1993). Valores iguales o superiores a $\rho=$ .70 son considerados aceptables (Nunnally, 1978). Con base en los objetivos y análisis propuestos en este trabajo, la presentación de los datos se desarrolló en cuatro fases, en las primeras tres se reportan los resultados psicométricos de las diferentes escalas adaptadas, mientras que en la cuarta fase se desarrolla el estudio de validez entre los diferentes constructos. 


\section{Resultados}

Se realizó una traducción (método directo) de los ítems de las diferentes escalas. Seis expertos en lengua inglesa, dos por cada instrumento, tradujeron los ítems del idioma original al español. Luego las diferentes versiones fueron comparadas y se realizaron los ajustes idiomáticos pertinentes procurando mantener cierta equivalencia conceptual, semántica y funcional (Mimura y Griffiths, 2008). En este punto, en la versión original de la escala de Metas de Progreso Académicas dos ítems referían a dos actividades cognitivas diferentes en el mismo enunciado ("Alcanzar y mantener notas altas en todas las materias" y "Aprender y entender el material de estudio de todas mis materias"). Por tanto, se decidió realizar una entrevista cognitiva con seis estudiantes de distintas carreras de la UNC, con el objetivo de indagar si las actividades mencionadas en el mismo enunciado eran similares (o no), y evitar así dificultades en la compresión y posterior respuesta. Se observó que dichas expresiones provocaron cierta ambigüedad debido a que los estudiantes diferencian entre las acciones de alcanzar (como un logro del momento) y mantener (como un esfuerzo constante), y de manera similar, aprender (asociado a la memorización y una postura acrítica ante el contenido) y entender (reviste un carácter más activo del sujeto en la adquisición del conocimiento). Con base en estos resultados se decidió subdividir los enunciados originales en dos, es decir; uno referido a "alcanzar notas altas" y otro a "mantener notas altas", y de forma similar con el siguiente ítem. De este modo, la escala de metas quedó conformada por 9 ítems.

\subsection{Fase 1. Expectativas académicas de resultado}

Preparación de los datos y análisis de ítems. Los casos perdidos oscilaron entre $2.4 \%$ (ítem 10) a 3.6\% (ítem 3). Al no superar el $5 \%$ establecido (Schafer, 1999) se procedió a imputar los datos mediante el modo de respuesta de cada participante. Respecto a la asimetría y curtosis, se observó que todos los ítems presentaron valores entre \pm 1.00 (ver tabla I). En cuanto a los casos atípicos, se identificó un caso univariado y 74 multivariados. Si bien la presencia de casos atípicos tiende a distorsionar los resultados, en este caso se decidió retenerlos con el fin de evitar la limitación en la generalización de los resultados, debido a que se dejaría de representar a un segmento de la población en estudio (Hair, Anderson, Tatham y Black, 1999).

Tabla I. Media, Desviación Estándar, Asimetría, Curtosis y pesos estandarizados de la escala Expectativas Académicas de Resultado

\begin{tabular}{ccccccc}
\hline & $M$ & DE & Asimetría & Curtosis & $\begin{array}{c}\beta \\
\text { Factor 1 }\end{array}$ & $\begin{array}{c}\beta \\
\text { Factor 2 }\end{array}$ \\
\hline ítem 1 & 5.08 & 1.59 & .46 & .26 & .79 & - \\
ítem 2 & 4.51 & 1.49 & .56 & .70 & .70 & - \\
ítem 3 & 4.24 & 1.71 & .49 & .52 & .65 & - \\
ítem 4 & 5.58 & 1.64 & .47 & -.45 & - & .84 \\
ítem 5 & 4.95 & 1.79 & .28 & .07 & - & .69 \\
ítem 6 & 4.62 & 1.83 & .22 & .12 & .66 & \\
ítem 7 & 5.33 & 1.85 & .26 & -.54 & - & .83 \\
ítem 8 & 4.27 & 1.64 & .60 & .34 & .76 & - \\
ítem 9 & 4.99 & 1.70 & .53 & -.13 & - & .83 \\
Ítem 10 & 4.80 & 1.67 & .43 & .13 & - & .78 \\
\hline
\end{tabular}

Nota: $M=$ media; $D E$ = desviación estándar; $\beta$ = peso beta estandarizado; Factor 1 = Expectativas académicas extrínsecas; Factor 2 = Expectativas académicas intrínsecas.

Análisis factorial confirmatorio. El ajuste a los datos no fue adecuado (CFI .915, TLI .887, RMSEA .201, 90\% CI 193.209 , WRMR 2.258). Los pesos de regresión estandarizados ( $p \leq .05)$ oscilaron entre .65 a .79 para el factor de expectativas extrínsecas, y entre .69 a .84 para expectativas intrínsecas. Debido a que el ajuste no fue óptimo según los criterios propuestos, se decidió inspeccionar los Índices de Modificación (IM). Cuando los residuos se encuentran altamente correlacionados se considera que existe redundancia debido a la superposición de contenidos (Brown, 2015). El análisis de los IM indicó una ganancia del ajuste al correlacionar los residuos entre los pares de ítems 1-2, 3-5, 3-6 y 5-6. Considerando que los pares de ítems refieren a un mismo tópico (oferta laboral, autopercepción y valoración) se decidió correlacionar los 
ítems. De este modo, el ajuste mejoró presentado un ajuste adecuado (CFI .973, TLI .959, RMSEA .121, 90\% $\mathrm{Cl} .112$.129, WRMR 1.244).

Consistencia interna. Los valores obtenidos fueron satisfactorios para ambas sub-escalas (expectativas extrínsecas, $\rho=.84$; expectativas intrínsecas $\rho=.90$ ).

\subsection{Fase 2. Metas de progreso académico}

Preparación de los datos y análisis de ítems. Los casos perdidos oscilaron entre $2 \%$ (ítem 9) a 2.8\% (ítem 4). Al no superar el $5 \%$ establecido se procedió a imputar los datos por el modo de respuesta de cada participante. Respecto a la asimetría y curtosis, se observó que todos los ítems presentaron valores entre \pm 1.00 (ver Tabla II). En cuanto a los casos atípicos, se identificaron 7 casos univariados y 26 casos multivariados, los cuales fueron conservados.

\section{Tabla II. Media, Desviación Estándar, Asimetría, Curtosis y pesos estandarizados de la escala Metas de Progreso Académicas}

\begin{tabular}{cccccc}
\hline & $\mathrm{M}$ & $\mathrm{DE}$ & Asimetría & Curtosis & $\beta$ \\
\hline ítem 1 & 3.70 & .89 & -.53 & -.04 & .69 \\
ítem 2 & 3.58 & .92 & -.41 & -.33 & .75 \\
ítem 3 & 3.53 & .92 & -.33 & -.28 & .80 \\
ítem 4 & 4.00 & .93 & -.75 & .17 & .48 \\
ítem 5 & 3.84 & .85 & -.48 & -.02 & .74 \\
ítem 6 & 3.32 & .98 & -.21 & -.29 & .74 \\
ítem 7 & 3.23 & .99 & -.21 & -.28 & .75 \\
ítem 8 & 3.55 & .90 & -.33 & -.35 & .76 \\
ítem 9 & 3.73 & .89 & -.49 & -.04 & .65 \\
\hline \multicolumn{7}{l}{ Nota: M = media; DE = desviación estándar; $\beta$ peso beta estandarizado. }
\end{tabular}

Análisis factorial confirmatorio. El ajuste a los datos fue adecuado (CFI .928, TLI .904, RMSEA .222, 90\% Cl .213 .231 , WRMR 3.992). Los pesos de regresión estandarizados ( $p \leq .05$ ) oscilaron entre .48 a .80. Debido a que el ajuste no fue óptimo se inspeccionaron los IM, los cuales indicaron una ganancia del ajuste al correlacionar los residuos entre los pares de ítems 6-7 y 8-9. Considerando que se trata de un solapamiento por la semejanza respecto al contenido, y con el fin de conservar los ítems originales del instrumento, se decidió correlacionar ambos pares de ítems. De este modo, el ajuste mejoró presentado un ajuste satisfactorio (CFI.993, TLI.990, RMSEA.072, 90\% Cl.063 .082, WRMR 1.142).

Consistencia interna. Se reportó un valor satisfactorio $(\rho=.89)$.

\subsection{Fase 3. Satisfacción académica}

Preparación de los datos y análisis de ítems. Los casos perdidos oscilaron entre $0.5 \%$ (ítem 6) a $0.9 \%$ (ítem 2). Al no superar el $5 \%$ establecido se procedió a imputar los datos por el modo de respuesta de cada participante. Respecto a la asimetría y curtosis, se observó que 5 ítems presentaron valores entre \pm 1.00 , un ítem presentó valor entre \pm 2.00 y un solo ítem presentó un valor > \pm 2.00 (ver tabla III). En cuanto a los casos atípicos, se identificaron 49 casos univariados y 29 multivariados, los cuales fueron conservados.

Análisis factorial confirmatorio. El ajuste a los datos fue satisfactorio (CFI .966, TLI.949, RMSEA .103, $90 \%$ Cl .091 .116 , WRMR 1.456). Los pesos de regresión estandarizados ( $p \leq .05)$ oscilaron entre .53 a .79.

Consistencia interna. Se reportó un valor satisfactorio $(\rho=.87)$. 
Tabla III. Media, Desviación Estándar, Asimetría, Curtosis y pesos estandarizados de la escala Satisfacción Académica

\begin{tabular}{cccccc}
\hline & $\mathrm{M}$ & $\mathrm{DE}$ & Asimetría & Curtosis & $\beta$ \\
\hline ítem 1 & 4.66 & .61 & -2.21 & 6.72 & .53 \\
ítem 2 & 4.00 & .83 & -.70 & .45 & .62 \\
ítem 3 & 3.97 & .81 & -.75 & .74 & .79 \\
ítem 4 & 3.98 & .86 & -.84 & .77 & .73 \\
ítem 5 & 3.75 & .85 & -.47 & .04 & .75 \\
ítem 6 & 4.20 & .80 & -.91 & .78 & .71 \\
Ítem 7 & 4.22 & .80 & -1.02 & 1.14 & .72 \\
\hline \multicolumn{5}{l}{ Nota: M = media; DE = desviación estándar; $\beta=$ peso beta estandarizado. }
\end{tabular}

\subsection{Fase 4. Estudio de validez}

Se obtuvo la matriz de correlaciones de las escalas Expectativas de Resultado, Metas de Progreso Académicas y Satisfacción Académica (ver tabla IV). Como se esperaba, tanto las expectativas de resultado extrínsecas e intrínsecas presentaron una fuerte correlación $(r=.76)$. Además, ambas medidas, es decir las consecuencias reforzantes positivas externas e internas, presentaron una correlación débil a moderada ( $r=.13$ y .20, respectivamente) con la percepción de bienestar de los estudiantes respecto al rol como estudiantes (satisfacción académica). Por su parte, aquellos estudiantes que perciben que están logrando un buen progreso en sus metas académicas indicaron encontrarse satisfechos con su rol de estudiantes $(r=.34)$. Si bien los resultados son consistentes con los reportados por la scct (Lent et al., 2007; Lent et al., 2013), los índices de correlación de este estudio fueron de menor magnitud, y además, no fue posible replicar la relación entre las Expectativas de Resultado y las Metas de Progreso Académicas.

Tabla IV. Matriz de correlaciones entre las escalas de Expectativas de Resultado, Metas de Progreso Académicas y Satisfacción Académica

\begin{tabular}{lcccc}
\hline & 1 & 2 & 3 & 4 \\
\hline 1. Expectativas extrínsecas & 1 & $.76^{* *}$ & .04 & $.13^{* *}$ \\
2. Expectativas intrínsecas & & 1 & .04 & $.20^{* *}$ \\
3. Metas de Progreso Académicas & & 1 & $.34^{* *}$ \\
4. Satisfacción Académica & & & 1 \\
\hline$* * p \leq .01$. & & &
\end{tabular}

\section{Discusión}

El objetivo de este trabajo fue adaptar las escalas de Expectativas de Resultado, Metas de Progreso y Satisfacción Académica en una población de estudiantes universitarios. La importancia de estos constructos radica en que permiten establecer posibles consecuencias producto de involucrarse en una actividad, orientar el comportamiento hacia fines específicos y revelar el nivel de bienestar que los estudiantes poseen en sus respectivas carreras, respectivamente; actitudes que contribuyen a explicar si los estudiantes continuarán sus estudios de grado (Lent et al., 1994).

En líneas generales, las escalas presentaron adecuadas propiedades psicométricas. Los índices arrojados por los estudios de consistencia interna fueron similares a los reportados por los trabajos originales. Los resultados del análisis factorial confirmatorio lograron replicar la estructura original de un factor de las escalas de Metas de Progreso Académicas y Satisfacción Académica, y de dos factores de la escala de Expectativas de Resultado. Si bien estos resultados sugieren que las escalas propuestas son viables en una muestra de estudiantes universitarios argentinos, es importante realizar nuevos estudios de validez de contenido. 
Respecto al estudio de validez, si bien las correlaciones observadas entre las diferentes escalas fueron las esperadas, la magnitud de las mismas fue débil a moderada. Por otro lado, como indica Lent et al. (1994), aquellos estudiantes que poseen expectativas positivas producto de un evento determinado (por ejemplo, graduarse de la universidad) tenderán a establecerse metas más exigentes. Sin embargo, los resultados de este estudio y los expuestos por Lent et al. (2007) no apoyaron dicha hipótesis. Una posible explicación podría deberse al hecho que las expectativas enunciadas en la escala no representen fielmente las creencias de los estudiantes respecto a lo que podría llegar a suceder una vez graduados. Otra posibilidad radicaría en que los estudiantes ven a muy largo plazo las consecuencias, siendo oportuno incluir medidas más próximas (previas a la graduación, por ejemplo). Lent y Brown (2006) llaman la atención respecto a que gran parte de los enunciados de las escalas de expectativas se centran en resultados positivos, siendo importante examinar cómo la anticipación de posibles consecuencias negativas ayudaría a explicar un comportamiento más determinado a ciertas metas.

A pesar que en este trabajo se realizaron entrevistas cognitivas para dilucidar la compresión de los ítems de la escala de expectativas, sería importante replicar este procedimiento para las escalas de metas y satisfacción académica. Esto se debe a que los test psicológicos se desarrollan en un contexto particular, por tanto, el uso de los mismos en un medio diferente al cual fue diseñado puede acarrear una serie de problemas, los cuales no se agotan en el proceso de adaptación. De este modo, las entrevistas indagan respecto a qué es lo que está pensado el sujeto y por qué responde de la manera que lo hace, actividades que permitirán corroborar la equivalencia semántica (refiere a que las palabras traducidas tengan el mismo significado) y funcional (que las acciones involucradas en los reactivos posean metas y dificultades semejantes en ambas culturas) de los ítems.

Respecto a las limitaciones del trabajo, se deben tomar en cuenta algunas consideraciones en relación con la muestra. Por un lado, la misma estuvo compuesta por un gran porcentaje de mujeres. Si bien no hay estudios que revelen diferencias en los constructos adaptados, sería oportuno equilibrar la cantidad de participantes en función del sexo en futuros estudios. Por otro lado, gran parte de los participantes se encontraba cursando carreras vinculadas al área de Ciencias de la Salud y Ciencias Humanas, y en menor proporción alumnos del área de Ciencias Naturales. Sería importante equiparar las áreas del conocimiento debido a que las expectativas, metas y satisfacción que persiguen y perciben los estudiantes puede llegar a variar de una carrera a otra (Lent et al., 2007).

A pesar del carácter preliminar de los resultados obtenidos, la adaptación de estas medidas genera nuevos instrumentos estandarizados al medio local, los cuales permiten un correcto registro y evaluación de las experiencias académicas de los estudiantes, variables sumamente relevantes en la predicción de la persistencia académica. Además, permitirá evaluar y diseñar estrategias de intervención, por ejemplo, counseling (asesoramiento) y gabinetes de seguimiento con el fin de identificar a aquellos jóvenes más propensos (bajas expectativas y nivel de satisfacción) a abandonar su carrera universitaria.

\section{Referencias}

Bandura, A. (1987). Pensamiento y Acción. Barcelona: Martínez Roca.

Bandura, A. (1997). Self-efficacy: The excercise of control. Nueva York: Freeman.

Brown, T. A. (2015). Confirmatory factor analysis for applied research. Nueva York: Guilford Publications.

Cortina, J. M. (1993). What is coefficient alpha? An examination of theory and applications. Journal of Applied Psychology, 78(1), 98.

Duffy, R. D. y Lent, R. W. (2009). Test of a social cognitive model of work satisfaction in teachers. Journal of Vocational Behavior, 75(2), 212-223. doi:10.1016/j.jvb.2009.06.001 
Flora, D. B. y Curran, P. J. (2004). An empirical evaluation of alternative methods of estimation for confirmatory factor analysis with ordinal data. Psychological Methods, 9(4), 466-491. doi:10.1037/1082989X.9.4.466

Flores, L. Y., Navarro, R. L., y Ali, S. R. (2016). The state of scct research in relation to social class future directions. Journal of Career Assessment, 25(1),6-23. doi:10.1177/1069072716658649

Fouad, N. A. y Guillen, A. (2006). Outcome expectations: Looking to the past and potential future. Journal of Career Assessment, 14(1), 130-142. doi: 10.1177/1069072705281370

Fouad, N. A. y Smith, P. L. (1996). A test of a social cognitive model for middle school students: Math and science. Journal of Counseling Psychology, 43, 338-346.

García de Fanelli, A. (2011). La educación superior en Argentina. En Educación Superior en Iberoamérica. Informe 2011. Santiago de Chile: CINDAUNIVERSIA.

García de Fanelli, A. (2013). Graduación y equidad en las universidades argentinas. Ponencia presentada en el VII Encuentro Nacional y IV Latinoamericano: La Universidad como objeto de investigación, 29-31 de agosto, Universidad Nacional de San Luis. Argentina.

George. D. y Mallery. P. (2011). IBM SPSS Statistics 21 step by step: A simple guide and reference. Boston, MA: Pearson Education.

Hair, J., Anderson, R., Tatham, R. y Black, W. (1999). Análisis multivariante (4a. edición). España: Prentice Hall.

Instituto Internacional para la Educación Superior en América y el Caribe (2006). Informe sobre la Educación Superior en América Latina y el Caribe 2000-2005. La Metamorfosis de la Educación Superior. Venezuela: UNESCO.

Lent, R. W. (2004). Toward a unifying theoretical and practical perspective on well-being and psychosocial adjustment. Journal of Counseling Psychology, 51(4), 482-509.

Lent, R. W. (2005). A social cognitive view of career development and counseling. En S. D. Brown y R. W. Lent (Eds.), Career development and counseling: Putting theory and research to work (pp. 101-127). Nueva York: John Wiley.

Lent, R. W. y Brown, S. D. (2006). On conceptualizing and assessing social cognitive constructs in career research: A measurement guide. Journal of career assessment, 14(1), 12-35.

doi:10.1177/1069072705281364

Lent, R. W., Brown, S. D. y Hackett, G. (1994). Toward a unifying social cognitive theory of career and academic interest, choice, and performance. Journal of Vocational Behavior, 45(1), 79-122.

Lent, R. W., Miller, M. J., Smith, P. E., Watford, B. A., Lim, R. H., Hui, K., Morrison, M. A., Wilkins, G. y Williams, K. (2013). Social cognitive predictors of adjustment to engineering majors across gender and race/ethnicity. Journal of Vocational Behavior, 83(1), 22-30. doi:10.1016/j.jvb.2013.02.006

Lent, R. W., Singley, D., Sheu, H., Gainor, K., Brenner, B. R., y Treistman, D. (2005). Social cognitive predictors of domain and life satisfaction: Exploring the theoretical precursors of subjective well-being. Journal of Counseling Psychology, 52, 429-442. doi:10.1037/0022-0167.52.3.429

Lent, R. W., Singley, D., Sheu, H., Schmidt, J. A., y Schmidt, L. C. (2007). Relation of social-cognitive factors to academic satisfaction in engineering students. Journal of Career Assessment, 15, 87-97.

doi:10.1177/1069072706294518 
Lent, R. W., Taveira, M., Sheu, H. y Singley, D. (2009). Social cognitive predictors of academic adjustment and life satisfaction in Portuguese college students: A longitudinal analysis. Journal of Vocational Behavior, 74, 190-198. doi:10.1016/j.jvb.2008.12.006

Lent, R. W. y Brown, S. (2008). Social cognitive career theory and subjective well-being in the context of work. Journal of Career Assessment, 16(1), 6-21. doi:10.1177/1069072707305769

Lent, R. W. y Brown, S. D. (2013). Social cognitive model of career self-management: Toward a unifying viewvof adaptive career behavior across the life span. Journal of Counseling Psychology, 60, 557-568. doi:10.1037/a0033446

Lent, R. W. y Brown, S. D. (2006). Integrating person and situation perspectives on work satisfaction: A social-cognitive view. Journal of Vocational Behavior, 69(2), 236-247. doi:10.1016/j.jvb.2006.02.006

Lent. R. W., Brown. S. D., Sheu. L. B., Gloster. C. S., Wilkins. G., Schmidt. L. C., Lyons. H. y Treistman. D. (2005). Social cognitive predictors of academic interests and goals in engineering: Utility for women and students at historically black universities. Journal of Counseling Psychology, 52. 84-92.

Maddux J.E. (2012). Self-efficacy: The power of believing you can. En C. R. Snyder y S. J. Lopez (Eds.), Handbook of Positive Psychology (pp. 277-287). Nueva York: Oxford University Press.

doi:10.1093/oxfordhb/9780195187243.013.0031

Mimura, C. y Griffiths, P. (2008). A japanese version of the perceived stress scale: cross-cultural translation and equivalence assessment. BMC psychiatry, 8(1), 85. doi:10.1186/1471-244X-8-85

Nunnally, J. C. (1978). Psychometric theory (2nd ed.). New York, NY: McGraw-Hill

Parrino, M. del C. (2012). ¿Evasión o expulsión? Los mecanismos de deserción en el primer año universitario. (Tesis inédita de doctorado), Programa Interuniversitario Doctorado en Educación.

Rottinghaus, P. J., Larson, L. M. y Borgen, F. H. (2003). The relation of self-efficacy and interests: a metaanalysis of 60 samples. Journal of Vocational Behavior, 62(2), 221-236.

Schafer, J. L. (1999). Multiple imputation: a primer. Statistical Methods in Medical Research, 8, 3-15.

Secretaria de Políticas Universitarias-Ministerio de Educación (2010). Anuario 2010. Estadísticas Universitarias. Recuperado de https://goo.gl/zSF03m

Sheu, H. B. y Bordon, J. J. (2016). scct research in the international context empirical evidence, future directions, and practical implications. Journal of Career Assessment, 25(1), 58-74.

doi:10.1177/1069072716657826

Tabachnick. B. G. y Fidell, L. S. (2011). Using multivariate statistics (6a. ed.). Needham Heights, MA: Pearson.

Vroom, V. H. (1964). Work and motivation. Nueva York: John Wiley.

Yu, C. Y. y Muthen, B. (2002, April). Evaluation of model fit indices for latent variable models with categorical and continuous outcomes. Documento presebrado en la Annual meeting of the American Educational Research Association, Nueva Orleans, LA. 